\title{
Rapid renal function decline is associated with an increased risk of cardiovascular complications
}

A lthough chronic kidney disease is a known risk factor for cardiovascular disease, little is known about whether sequential changes in estimated glomerular filtration rate (eGFR) have prognostic importance. Two studies published in the same issue of the Journal of the American Society of Nephrology have now found, however, that a decline in renal function is indeed associated with an increased risk of cardiovascular complications.

Kunihiro Matsushita and co-workers used data from 13,029 individuals (aged 45-64 years) in the US population-based Atherosclerosis Risk in Communities (ARIC) study to investigate whether 3-year and 9-year changes in eGFR are associated with risk of coronary heart disease and allcause mortality. Participants underwent clinical examinations on enrollment and at 3 -year intervals for 9 years.

The researchers stratified ARIC participants into quartiles according to their percentage annual change in eGFR at 3 years and 9 years. Using Cox proportional hazards models adjusted for covariates including baseline eGFR, they found that individuals with the greatest annual declines in eGFR (quartile 1: annual eGFR decline $-52.76 \%$ to $-5.65 \%$ ) had a $30 \%$ higher risk of incident coronary heart disease and a $22 \%$ higher risk of allcause mortality at 3 years than individuals with relatively stable eGFRs (annual declines in eGFR of between $-0.47 \%$ and $-0.33 \%)$. Findings were similar at 9 years. In addition, a rapid decline in kidney function was associated with an increased risk of all-cause mortality independently of renal function at follow-up. "Our findings suggest that the change in eGFR provides additional prognostic information to a single eGFR measurement," states Matsushita. "Therefore, physicians should pay attention to the trend in eGFR in addition to the latest eGFR."

In the second study, Michael Shlipak et al. investigated whether changes in renal function over a 7 -year period were

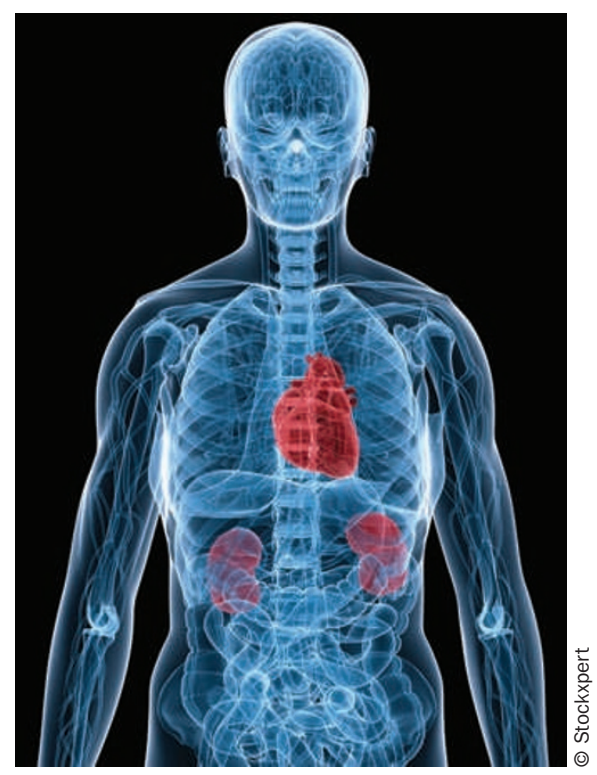

associated with the incidence of heart failure, myocardial infarction, stroke and peripheral artery disease during the subsequent 8 years among the elderly individuals enrolled in the communitybased Cardiovascular Health Study. "We did this paper to evaluate if the change in kidney function over the prior 7 years was important above and beyond the actual level of current function," notes Shlipak.

The Cardiovascular Health Study was designed to evaluate risk factors for cardiovascular disease in individuals aged $\geq 65$ years. Renal function was measured at baseline and after 3 years and 7 years of follow-up. Repeated measurements of serum creatinine and cystatin C levels were available for 4,380 participants. Shlipak et al. found that a rapid decline in cystatin-C-based eGFR-defined as a decline of $>3 \mathrm{ml} / \mathrm{min} / 1.72 \mathrm{~m}^{2}$ per year-occurred in 1,083 participants (25\%). The researchers found that the incidences of heart failure, myocardial infarction, stroke and peripheral artery disease were all significantly higher in patients who had experienced a rapid decline in cystatin-Cbased eGFR than in those without rapid decline. In multivariate analyses adjusted for demographic characteristics and cardiovascular risk factors, individuals who had shown rapid decline in kidney function had an increased risk of heart failure of about $30 \%$, an approximately $40 \%$ increased risk of myocardial infarction, and an approximately $60 \%$ increased risk of peripheral arterial disease, although this last finding was not statistically significant. The researchers found no significantly increased risk of stroke in individuals with a rapid decline in renal function. Rapidly declining renal function was independently associated with an increased risk of myocardial infarction and peripheral arterial disease, even among individuals without chronic kidney disease at final follow-up.

In analyses of creatinine-based eGFRs, rapid decline was associated only with an increased risk of incident heart failure. "It is critical that we used cystatin $C$ rather than creatinine to study trajectories in kidney function in the elderly, as cystatin C is far less influenced by the unpredictable body composition of elderly persons," explains Shlipak.

Shlipak concludes that "...it [the study] is a step closer toward proving that kidney health does drive the risk of cardiovascular disease. Clinically, it reminds us to not only look at today's kidney function, but also consider the recent trend in forecasting the likelihood of both progressive kidney disease and cardiovascular disease."

The findings from these two studies indicate that a patient's change in renal function over time, as well as their current level of renal function, provides important information about their risk of cardiovascular complications.

Rebecca Ireland

Original articles Matsushita, K. et al. Change in estimated GFR associates with coronary heart disease and mortality. J. Am. Soc. Nephrol. 20, 2617-2624 (2009) | Shlipak, M. G. et al. Rapid decline of kidney function increases cardiovascular risk in the elderly. J.Am. Soc. Nephrol. 20, 2625-2630 (2009) 